\title{
Post Transplant Anaemia
}

\author{
Bhadran Bose and David Mudge \\ Princess Alexandra Hospital, Brisbane \\ Australia
}

\section{Introduction}

Anaemia, as a complication of end stage kidney disease (ESKD), is well studied (Eschbach and Adamson 1985). Reports indicate that anaemia in this patient population is associated with numerous morbidities, but predominantly cardiovascular complications such as left ventricular hypertrophy and heart failure (Wizemann, Schafer et al. 1993; Harnett, Foley et al. 1995). Contrary to the ample data available regarding anaemia in ESKD population, much less is known about the epidemiology of post-transplant anaemia (PTA), and only few studies have systematically investigated this issue (Saito, Fujiwara et al. 1998; Yorgin, Scandling et al. 2002; Mix, Kazmi et al. 2003; Vanrenterghem, Ponticelli et al. 2003).

A successful renal allograft will correct not only the excretory functions of the kidney but also the endocrine functions (through the restored synthesis of erythropoietin and vitamin D) including the correction of anaemia. However, in the majority of transplant recipients, the renal graft does not function optimally. It is well known that renal excretory functions are not restored completely, but the extent to which renal endocrine functions are restored is only recently becoming better understood. A recent European survey of ten transplant centres revealed a prevalence of PTA as high as 42\% (Molnar et al, 2011).

PTA may occur at any time after renal transplantation. Early PTA (from the time of surgery until 3 months post-operatively) is most likely to be related to pre-transplant anaemia of ESKD, the surgery itself, iron deficiency, immunosuppression, inflammation or infection (Miles, Markell et al. 1997; Turkowski-Duhem, Kamar et al. 2005) or some combination of these. Later PTA is more likely to be related to consequences of long-term immunosuppression (particularly anti-metabolite medications) or a failing allograft. All the published large scale studies to date have focused mainly on late PTA (Miles, Markell et al. 1997; Vanrenterghem, Ponticelli et al. 2003; Turkowski-Duhem, Kamar et al. 2005), and there are a lack of studies looking at the management of early PTA, in particular, the optimal management of iron deficiency at the time of kidney transplantation.

The importance of PTA is that it is one of the commonest complications after kidney transplantation, that it is strongly associated with cardiovascular morbidity (the primary cause of death of kidney transplant recipients, accounting for approximately one half of such deaths), and that its optimal treatment, either with iron, erythropoietin, or other agents, has yet to be determined by large-scale randomised controlled trials.

\section{Prevalence}

PTA remains a common complication after kidney transplantation, with an incidence ranging from nearly $80 \%$ at day 0 to as low as $25 \%$ at 1 year (Saito, Fujiwara et al. 1998; 
Yorgin, Scandling et al. 2002; Mix, Kazmi et al. 2003; Vanrenterghem, Ponticelli et al. 2003). To date, there are very few published studies of PTA, and very few presenting data on interventions for PTA.

One of the largest studies (Vanrenterghem, Ponticelli et al. 2003) was published in the American Journal of Transplantation, and included 4,263 kidney transplant patients from 72 centres in 16 European countries (TRansplant European Survey on Anaemia Management [TRESAM] survey). The patients were divided into four cohorts and assessed at 6 months $(n=1003), 1$ year $(n=960), 3$ years $(n=1254)$ and 5 years $(n=1046)$ after kidney transplantation. Recipients of multiple organs, pregnant women and children aged $<10$ years were excluded from the study.

Demographic data indicate that the patients included in the TRESAM survey were representative of the European transplant population. Approximately $60 \%$ of patients were male and $40 \%$ were female, and these proportions were similar in the four cohorts. The mean (SD) age of transplant recipients at transplantation was 45.5 (13.1) years, although patients who had received their transplant more recently were significantly $(p=0.01)$ older at transplantation than patients who had received their transplant 5 years ago. The proportion of patients in this survey who had received their kidney from a living donor was relatively low $(\sim 10 \%)$. The most prevalent underlying kidney disease in the TRESAM survey was chronic glomerulonephritis (29.8-37.0\% across the four cohorts). The prevalence of diabetic nephropathy was uncharacteristically low $(6.5-7.5 \%)$ by contemporary standards. The most frequently occurring co-morbidities were coronary artery disease (13.0-16.1\%), hepatitis B/C (9.3-10.8\%) and type 2 diabetes mellitus (8.7-10.1\%).

Pre-transplant haemoglobin $(\mathrm{Hb})$ concentrations in one study were significantly higher in patients who had received their transplant more recently than in patients who had received their transplant 5 years earlier, suggesting progressive anaemia with time. In that study, the mean $\mathrm{Hb}$ concentrations were $11.9 \mathrm{~g} / \mathrm{dL}$ in the 6 month cohort $v s 10.8 \mathrm{~g} / \mathrm{dL}$ in the 5 year cohort ( $\mathrm{p}<0.01$; Eschbach and Adamson, 1985)

Anaemia, defined as $\mathrm{Hb}$ concentration $\leq 13 \mathrm{~g} / \mathrm{dL}$ for male patients and $\leq 12 \mathrm{~g} / \mathrm{dL}$ for female patients, was present in $38.6 \%$ of patients overall in this survey, and was equally distributed between the sexes. Of these patients, $11.6 \%$ had moderate anaemia ( $\mathrm{Hb}$ concentration $>11$ and $\leq 12 \mathrm{~g} / \mathrm{dL}$ for male patients and $>10$ and $\leq 11 \mathrm{~g} / \mathrm{dL}$ for female patients), while $8.5 \%$ had severe anaemia $(\mathrm{Hb}$ concentrations $\leq 11 \mathrm{~g} / \mathrm{dL}$ for male patients and $\leq 10 \mathrm{~g} / \mathrm{dL}$ for female patients). In patients with a serum creatinine levels $>2 \mathrm{mg} / \mathrm{dL}, 60.1 \%$ were considered anaemic, whereas in patients with a serum creatinine level $\leq 2 \mathrm{mg} / \mathrm{dL}$, only $29.0 \%$ of patients were considered anaemic $(\mathrm{p}<0.01)$. The findings of the TRESAM survey were in agreement with the results published by a contemporary American group (Yorgin, Scandling et al. 2002). The aforementioned 5-year follow up of the TRESAM survey (Molnar et al, 2011) reported similar rates of anaemia at $42 \%$ overall, suggesting that the problem is not improving over time despite awareness of the problem.

The group from Boston, MA undertook a retrospective longitudinal cohort study (Mix, Kazmi et al. 2003) to characterise changes in haematocrit (Hct) following kidney transplantation and to identify factors associated with those changes. The study included 240 patients who underwent kidney transplantation in Tufts-New England Medical Centre, Boston, MA. Hct levels were available for $94 \%$ of patients at 1-month follow-up and for 95$100 \%$ of patients at subsequent follow-up intervals. At the time of transplant surgery, $22 \%$ $(95 \%$ CI $16 \%, 28 \%)$ of the cohort had Hct $<30 \%$. The mean Hct rose from its nadir of 33\% (95\% CI 27\%. 39\%), both at the time of transplantation and at 1 month after transplantation, 
to a peak of $40 \%(95 \%$ CI 34\%, 46\%) 12 months after transplantation, and declined thereafter. During the first year after transplantation, the proportion of patients with Hct $<36 \%$ steadily decreased from $76 \%(95 \%$ CI $70 \%, 82 \%)$ at transplantation to $21 \%(95 \%$ CI $15 \%, 27 \%)$ at 12 months after transplantation. The proportion of patients with Hct $<33 \%$ declined over a shorter time interval, from $48 \%$ (95\% CI 42\%, 54\%) at transplantation to $7 \%$ (95\% CI 3\%, $11 \%)$ at 6 months after transplantation. These data closely reflect the European experience as previously described.

\section{Risk factors for PTA}

In the post-transplant setting anaemia may be contributed to by blood loss, iron deficiency, bone marrow suppression by anti-proliferative agents (mycophenolate, azathioprine), erythropoietin deficiency, and inflammation (Table 1). The relative contributions of these components may be difficult to ascertain in an individual patient and may often be due to a combination of these factors.

\begin{tabular}{ll}
\hline Early PTA & \multicolumn{1}{c}{ Late PTA } \\
\hline Immunosuppressive agents & $\downarrow$ red cell survival \\
Surgical blood loss & Immunosuppressive agents \\
Frequent blood sampling & $\downarrow$ erythrocyte production \\
Drugs-ACEi/ARB & Low excretory $+/$ - endocrine graft function \\
Iron deficiency & EPO resistance \\
Delayed graft function & \\
Persistent uraemic toxins & \\
\hline
\end{tabular}

$\mathrm{ACEi}=$ Angiotensin converting enzyme inhibitor, $\mathrm{ARB}=$ Angiotensin Receptor Blocker. EPO resistance is defined as the need of $\geq 300 \mathrm{IU} / \mathrm{Kg} /$ week.

Table 1. Common risk factors for PTA

\subsection{Early PTA}

A prospective study (Turkowski-Duhem, Kamar et al. 2005) published in Transplantation attempted to identify independent predictors of anaemia at 6 months and 12 months posttransplant. The authors studied 99 patients who had undergone kidney transplantation at Toulouse University Hospital between January 2001 and January 2002. Anaemia as defined by WHO criteria was observed in $38.6 \%$ of patients, which is a very similar rate to that found in other published studies from the same era.

Immunosuppressive treatment, particularly with antimetabolite drugs, is one of the most likely contributors to PTA. The purine synthesis inhibitors, azathioprine and mycophenolate mofetil (MMF), are known to cause anaemia (Gossmann, Kachel et al. 1993; de Sevaux, Hilbrands et al. 1998; Kuypers, Claes et al. 2004; Shipkova, Spielbauer et al. 2004). Macrocytosis was present in the majority of the patients in those studies treated with azathioprine. Mammalian target of rapamycin (mTOR) inhibitors (e.g., sirolimus) are also associated with PTA, and particularly with microcytosis even in the absence of iron deficiency (Thaunat, Beaumont et al. 2007). In that study nearly all patient received concomitant MMF and for the $42.3 \%$ who received induction therapy with rabbit antithymocyte globulin (ATG), the incidence of PTA was lower than previously reported values, at only $25 \%$ at 1 year post-transplantation. This could merely reflect selection bias. 
Some recent report suggests that sirolimus-based therapy was an independent factor of PTA (Augustine, Knauss et al. 2004). For example, the prevalence of PTA was 31\% with MMFbased immunosuppression and as high as $57 \%$ with sirolimus-based immunosuppression. Through mTOR inhibition, sirolimus blocks S6 kinase activity and consequently impairs cell replication in an erythroid cell line (Jaster, Bittorf et al. 1996) leading to anaemia.

One other obvious contributor to post-transplant anaemia is iron deficiency. Iron deficiency, defined by low ferritin and a low transferrin saturation, was present in $17 \%$ (Mahmud, Aziz et al. 2002) and $34.6 \%$ (Kim, Park et al. 2003) of anaemic patients in the post-transplant setting. Patients coming to kidney transplantation are known to be frequently iron deficient both in absolute and functional terms (Lorenz, Kletzmayr et al. 2002).

Many studies have demonstrated that the occurrence of PTA is related to renal allograft function, with declining function related to worsening anaemia as is seen in the chronic kidney disease (CKD) population. The results of the TRESAM survey showed a strong correlation between $\mathrm{Hb}$ levels and graft function expressed as serum creatinine and creatinine clearance: the majority of anaemic patients had creatinine levels $>2 \mathrm{mg} / \mathrm{dL}$ (Vanrenterghem, Ponticelli et al. 2003). Turkowski-Duhem found an effect of post-transplant renal function on haemoglobin levels at 6 months. Both delayed graft function (DGF) and renal allograft function, defined by creatinine clearance, were independent factors associated with the occurrence of anaemia at 12 months post transplantation(TurkowskiDuhem, Kamar et al. 2005). In another study it was reported that at both 6 and 12 months post transplantation higher serum creatinine values (defined as each $1 \mathrm{mg} / \mathrm{dL}$ increase in serum creatinine level) were significant independent risk factors for anaemia (Shibagaki and Shetty 2004). Similarly, in a further study (Yorgin, Scandling et al. 2002) serum $\mathrm{CO}_{2}$, blood urea nitrogen (BUN) and creatinine correlated with anaemia at 1 year post transplantation (Yorgin, Scandling et al. 2002). These findings were explained by the decreased production of erythropoietin (Kuypers, Claes et al.) and also the increase in erythropoietin (EPO) resistance relative to the decline in renal excretory function in the early 1990's. (van Dullemen, Luykx-de Bakker et al. 1992).

In the TRESAM survey, transplant recipients who had experienced one or more rejection episodes, or who had received a second or third graft, had lower haemoglobin levels than recipients without rejection episodes or recipients with a first transplant. An analysis using DNA microarrays identified a cluster of genes related to haemoglobin synthesis and/or erythropoiesis that was altered in kidneys with renal allograft rejection compared with normal allograft kidneys (Chua, Barry et al. 2003).

Another factor relevant to early PTA is cytomegalovirus (CMV) infection. TurkowskiDurhem showed on univariate analysis that at 12 months post transplantation, the prevalence of CMV seropositivity was significantly higher in those presenting with anaemia (78\%) than in those without anaemia (51\%), suggesting CMV infection might be a predisposing factor to PTA. However, on multivariate analysis, CMV status was no longer a significant predictor. . Mix et al. found that positive CMV donor status and negative recipient status (CMV D+/R-) was a factor independently associated with a greater likelihood of PTA, in this case defined as a haematocrit (Hct) $<36 \%$ at 6 months post transplantation (Mix, Kazmi et al. 2003). These two observations suggest that CMV infection itself, and/or the use of drugs to prevent or treat CMV infection/disease, might result in a degree of bone-marrow suppression leading to PTA.

Angiotensin converting enzyme (ACE) inhibitor therapy is known to induce anaemia (Gossmann, Kachel et al. 1993), and has been used to treat post-transplant erythrocytosis 
(Rostaing, Boisseau et al. 1995). The TRESAM survey reported that the use of ACE inhibitors or angiotensin II receptor blockers (ARB's) was associated with a higher odds ratio for anaemia $(\mathrm{OR}=1.55)$; they also found that it increased the risk of severe anaemia. Winckelmayer et al. observed that patients taking ACE inhibitors had significantly lower Hct levels compared with patients not taking them. In addition, a significant curvilinear dose-response relationship was found between ACE inhibitor dose and Hct level.

Turkowski-Durhem also observed that the platelet count at day-7 post transplantation was an independent predictive factor for anaemia at both 6 months (OR $0.12(0.03-0.48)$; $\mathrm{P}=0.002)$ and 12 months (OR 0.096 (0.01 -1.04); $\mathrm{P}=0.05)$, i.e. more anaemic patients had a significantly lower platelet count $(<200 \times 10 \% / \mathrm{L})$ than non-anaemic patients $(72 \% \mathrm{vs} .38 \%$ at 6 months, and $65 \%$ vs. $45 \%$ at 12 months). The significance of the association with platelet counts at day-7 post transplant, and the occurrence of PTA at 6 and 12 months, persists even after excluding patients that received induction therapy with ATG because the latter can result in thrombocytopenia. There was no explanation given for this observation, although it could just represent a different measure of overall bone marrow suppression

The other causes of early PTA have been attributed to blood loss at the time of surgery, frequent blood sampling and the persistent effect of uraemic toxins.

\subsection{Late PTA}

PTA that occurs late after transplantation has been attributed to decreased red blood cell survival (Najean, Rain et al. 1997), erythrocyte hypoproduction due to iron deficiency (Teruel, Lamas et al. 1989; Beshara, Birgegard et al. 1997; Miles, Markell et al. 1997), low excretory graft function (Miles, Markell et al. 1997), and immunosuppressive agents (Solheim, Albrechtsen et al. 1987; Yoshimura, Oka et al. 1989; Creemers, van Boven et al. 1993; Zazgornik 1997).

A retrospective longitudinal analysis (Yorgin, Scandling et al. 2002) of adult renal transplant recipients was published in American Journal of Transplantation looking at the prevalence, severity and predictors of late PTA. The study population of adults ( $>18$ years) transplanted during 1995 at Stanford University $(n=88)$ and University of North Carolina $(n=40)$ were selected. Post-transplant anaemia was a common problem which affected $30 \%$ of the study population at least once during the first 5 years after transplant. Recurrence of PTA was common $(50 \%)$ and the prevalence and severity of anaemia increased with time after transplantation.

Renal excretory function is strongly correlated with anaemia in adult renal transplant recipients. Yet the effect of renal excretory function on anaemia is not absolute, as a small proportion of anaemic patients have creatinine clearance values $>75 \mathrm{~mL} / \mathrm{min} / 1.73 \mathrm{~m}^{2}$ (i.e. normal), whereas conversely, some non-anaemic patients have creatinine clearance $<50$ $\mathrm{mL} / \mathrm{min} 1.73 \mathrm{~m}^{2}$.(Yorgin, Scandling et al. 2002). A study by van Dullemen et al. demonstrated that EPO production decreases and EPO resistance increases proportionally to the decline in renal excretory function (van Dullemen, Luykx-de Bakker et al. 1992). Other factors not related to declining renal excretory function include DGF, acute tubulointerstitial rejection (Besarab, Caro et al. 1987; Heidenreich, Tepel et al. 1995), chronic rejection (Heidenreich, Tepel et al. 1995) and possibly long-term calcineurin inhibitor toxicity (Jensen, Hansen et al. 1994) may contribute to PTA by leading to diminished EPO production.

Since renal tubular injury may be attributable to DGF, allograft rejection and calcineurin inhibitor toxicity in renal transplant recipients, and that all of these complications are 
frequent, another factor which has been evaluated as an important indicator of renal tubular function is serum total $\mathrm{CO}_{2}$. In one study, Anaemic patients had lower serum total $\mathrm{CO}_{2}$ values ( $p=0.019$ at 1 year post transplant and $p<0.0001$ at 5 years post transplant) (Yorgin, Scandling et al. 2002). The authors speculate that the presence of metabolic acidosis is a surrogate indicator of renal tubular injury, diminished renal excretory function and diminished EPO production, however this hypothesis has not been tested.

Yorgin et al also found that few anaemic patients were ever treated with EPO or even iron therapy. This finding may demonstrate a need for increased awareness of anaemia and increased concern about subnormal Hct by the patient's primary nephrologists and transplant physicians.

\section{Treatment of PTA}

There is a paucity of good quality and large scale intervention trials for PTA in the transplant literature, although some registry studies and multi-centre surveys do shed some light on practice patterns. For example, in the TRESAM survey rates of ESA use were reported. Surprisingly, of the 3969 patients overall for whom the use of erythropoietic therapy was documented, only 207 (5.2\%) had received an erythropoietin-stimulating agent (ESA) for the treatment of anaemia. Even more striking was the fact that among the group of anaemic patients $(n=1539)$, only $10 \%$ had received an ESA or other specific therapy. Of the 343 patients with severe anaemia, $17.8 \%$ were treated with erythropoietic therapy (Fig 1).

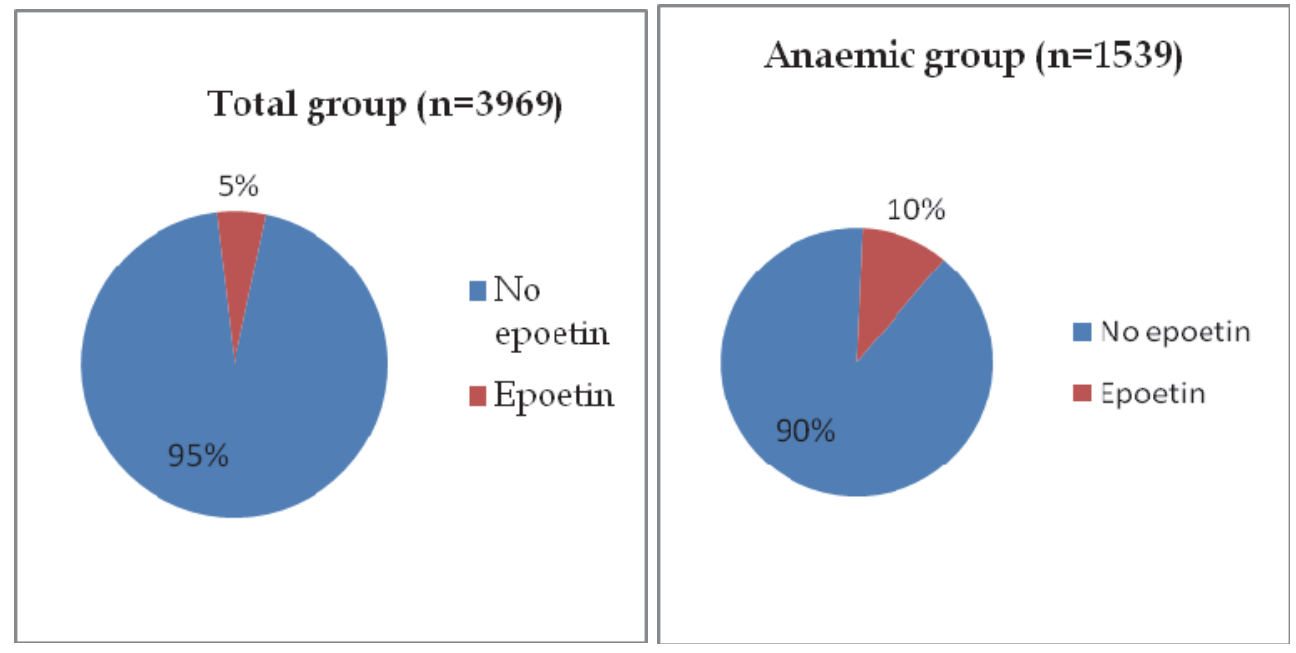

Fig. 1. Epoetin treatment in the TRESAM survey.

Possible reasons for the reluctance of nephrologists to correct anaemia in transplant patients include a relative paucity of published studies on intervention strategies for PTA, an underrecognition of the importance of the problem in terms of contribution to overall cardiovascular risk (also due to lack of specific studies demonstrating this) and finally the "psychological factor" of concerns regarding the efficacy and safety of therapy. In terms of safety of therapy, there have been concerns regarding the normalisation of $\mathrm{Hb}$ 
concentrations with ESA in the CKD population and. A few studies, involving small number of patients, have suggested that transplant recipients treated with epoetin develop hypertension, which requires treatment with anti-hypertensive drugs (Muirhead 1999; Kim, Park et al. 2003). However, to date, there have been no indications that patients treated with erythropoietic therapy after transplantation show a more rapid deterioration of their allograft function(Muirhead 1999).

\subsection{Erythropoietin-stimulating agent use}

Several small-scale studies suggest that ESA may be an effective treatment for posttransplant anaemia (Traindl, Barnas et al. 1994; Muirhead 1999; Kim, Park et al. 2003). The study done by Lezaic (Lezaic, Djukanovic et al. 1995) suggested that the response to erythropoietic therapy of patients with anaemia post-transplant is comparable with that of anaemia due to other forms of renal failure. As EPO resistance is common in these patients, they should be evaluated for secondary or tertiary hyperparathyroidism, hypothyroidism, haemolysis, and GI blood loss if a reticulocyte spike is not detected 7 days post transplantation.

Several recent studies in the CKD literature (Pfeffer et al. 2009, Drueke et al 2006) regarding haemoglobin targets have resulted in concern over the potential for increasing the risk of stroke and possibly malignancy-related death when haemoglobin targets of $>13 \mathrm{mg} / \mathrm{dL}$ are aimed for. The optimum $\mathrm{Hb}$ target for kidney transplant recipients remains unknown. Future trials will need to assess these endpoints in the post-transplant situation in order to establish the safety of ESA in this setting.

\subsection{Iron therapy}

Replacement of depleted iron stores are necessary for the correction of PTA. Functional iron deficiency is a common problem in CKD populations and may also be a problem in kidney transplant recipients. The best form of iron replacement in this setting is yet to be established. Oral iron is poorly absorbed, often not tolerated, and may interfere with the absorption of immunosuppressants. Intravenous iron has been used to replenish iron stores (Macdougall 1999) in transplant recipients as it has been in CKD patients in order to get around the problems of oral iron supplements. But a study currently in press but whose protocol was recently published (Mudge, Tan et al. 2009) showed that intravenous versus oral iron was not associated with a reduction in PTA, but did lead to a non-significant reduction in gastro-intestinal side-effects, numerically fewer blood transfusions and was equally efficacious in the treatment of post-transplant anaemia.

The measurement of iron status in post-kidney transplant patients, as in CKD patients, remains problematic. Existing measurements include serum iron, ferritin, and transferrin saturation. Ferritin is elevated in the setting of inflammation and therefore may not be indicative. New measures of iron status such as hepcidin show promise for more accurate estimation of iron status in this setting, but as yet a reference range has not been elucidated.

\subsection{Other treatments}

Other nutritional parameters such as B vitamins (B12, folate etc) are important in the pathogenesis of anaemia, and patients who have been treated with haemodialysis prior to transplantation are at risk of B-group vitamin deficiencies due to the removal of these water soluble vitamins with long-term dialysis, and especially with extended-hours dialysis 
(Coveney, 2010). Most such patients would be supplemented with B-group vitamins during their dialysis treatment however.

\section{Conclusions}

PTA is a common problem in kidney transplant recipients and further studies are needed to better determine the relative contributions of iron deficiency, EPO deficiency, and bone marrow suppression, with a view to better determining the optimum strategy for its correction in individual patients. Anaemia is a risk factor for cardiovascular events and may be a contributor to the high cardiovascular event rate seen in kidney transplant recipients.

\section{References}

Augustine, J. J., T. C. Knauss, et al. (2004). "Comparative effects of sirolimus and mycophenolate mofetil on erythropoiesis in kidney transplant patients." American journal of transplantation : official journal of the American Society of Transplantation and the American Society of Transplant Surgeons 4(12): 2001-2006.

Besarab, A., J. Caro, et al. (1987). "Dynamics of erythropoiesis following renal transplantation." Kidney international 32(4): 526-536.

Beshara, S., G. Birgegard, et al. (1997). "Assessment of erythropoiesis following renal transplantation." European journal of haematology 58(3): 167-173.

Chua, M. S., C. Barry, et al. (2003). "Molecular profiling of anemia in acute renal allograft rejection using DNA microarrays." American journal of transplantation : official journal of the American Society of Transplantation and the American Society of Transplant Surgeons 3(1): 17-22.

Coveney, N., Polkinghorne, K.R., Linehan, L. et al. (2010). "Water-soluble vitamin levels in extended hors hemodialysis." Hemodialysis International Nov 24. doi: 10.1111/j.1542-4758.2010.00505.x. [Epub ahead of print]

Creemers, G. J., W. P. van Boven, et al. (1993). "Azathioprine-associated pure red cell aplasia." Journal of internal medicine 233(1): 85-87.

de Sevaux, R. G., L. B. Hilbrands, et al. (1998). "A randomised, prospective study on the conversion from cyclosporine-prednisone to cyclosporine-azathioprine at 6 months after renal transplantation." Transplant international : official journal of the European Society for Organ Transplantation 11 Suppl 1: S322-324.

Drueke, T.B. et al (2006). "Normalization of hemoglobin level in patients with chronic kidney disease and anemia." New England Journal of Medicine 355(20): 2071-2084.

Eschbach, J. W. and J. W. Adamson (1985). "Anemia of end-stage renal disease (ESRD)." Kidney international 28(1): 1-5.

Gossmann, J., H. G. Kachel, et al. (1993). "Anemia in renal transplant recipients caused by concomitant therapy with azathioprine and angiotensin-converting enzyme inhibitors." Transplantation 56(3): 585-589.

Harnett, J. D., R. N. Foley, et al. (1995). "Congestive heart failure in dialysis patients: prevalence, incidence, prognosis and risk factors." Kidney international 47(3): 884890.

Heidenreich, S., M. Tepel, et al. (1995). "Prognostic value of serum erythropoietin levels in late acute rejection of renal transplants." American journal of kidney diseases : the official journal of the National Kidney Foundation 25(5): 775-780. 
Jaster, R., T. Bittorf, et al. (1996). "Inhibition of proliferation but not erythroid differentiation of J2E cells by rapamycin." Biochemical pharmacology 51(9): 1181-1185.

Jensen, J. D., H. E. Hansen, et al. (1994). "Increased serum erythropoietin level during azathioprine treatment in renal transplant recipients." Nephron 67(3): 297-301.

Kim, H. C., S. B. Park, et al. (2003). "Anemia following renal transplantation." Transplantation proceedings 35(1): 302-303.

Kuypers, D. R., K. Claes, et al. (2004). "Clinical efficacy and toxicity profile of tacrolimus and mycophenolic acid in relation to combined long-term pharmacokinetics in de novo renal allograft recipients." Clinical pharmacology and therapeutics 75(5): 434-447.

Lezaic, V., L. Djukanovic, et al. (1995). "Recombinant human erythropoietin treatment of anemia in renal transplant patients." Renal failure 17(6): 705-714.

Lorenz, M., J. Kletzmayr, et al. (2002). "Anemia and iron deficiencies among long-term renal transplant recipients." Journal of the American Society of Nephrology : JASN 13(3): 794-797.

Macdougall, I. C. (1999). "Strategies for iron supplementation: oral versus intravenous." Kidney international. Supplement 69: S61-66.

Mahmud, S. N., R. Aziz, et al. (2002). "Anemia characteristics after renal transplantation." Transplantation proceedings 34(6): 2428.

Miles, A. M., M. S. Markell, et al. (1997). "Anemia following renal transplantation: erythropoietin response and iron deficiency." Clinical transplantation 11(4): 313315.

Mix, T. C., W. Kazmi, et al. (2003). "Anemia: a continuing problem following kidney transplantation." American journal of transplantation : official journal of the American Society of Transplantation and the American Society of Transplant Surgeons 3(11): 1426-1433.

Mudge, D. W., K. S. Tan, et al. (2009). "Intravenous versus oral iron supplementation for correction of post-transplant anaemia in renal transplant patients." BMC nephrology 10: 14.

Muirhead, N. (1999). "Erythropoietin and renal transplantation." Kidney international. Supplement 69: S86-92.

Najean, Y., J. D. Rain, et al. (1997). "Red cell and platelet kinetics in chronic cytopenias following transplantation." Hematology and cell therapy 39(5): 233-236.

Pfeffer, M.A., Burdmann, M.D., Chen, C.Y., et al. (2009). "A trial of darbepoetin alfa in type 2 diabetes and chronic kidney disease" New England Journal of Medicine 361(21): 2019-32

Rostaing, L., M. Boisseau, et al. (1995). "Correction of post-renal transplant erythrocytosis by enalapril." Scandinavian journal of urology and nephrology 29(4): 399-406.

Saito, S., T. Fujiwara, et al. (1998). "Anemia following renal transplantation." Transplantation proceedings 30(7): 3025-3026.

Shibagaki, Y. and A. Shetty (2004). "Anaemia is common after kidney transplantation, especially among African Americans." Nephrology, dialysis, transplantation : official publication of the European Dialysis and Transplant Association - European Renal Association 19(9): 2368-2373.

Shipkova, M., B. Spielbauer, et al. (2004). "cDNA microarray analysis reveals new candidate genes possibly linked to side effects under mycophenolate mofetil therapy." Transplantation 78(8): 1145-1152. 
Solheim, B. G., D. A. Albrechtsen, et al. (1987). "Hemolytic anemia in cyclosporine-treated recipients of kidney or heart grafts from donors with minor incompatibility for ABO antigens." Transplantation proceedings 19(5): 4236-4238.

Teruel, J. L., S. Lamas, et al. (1989). "Serum ferritin levels after renal transplantation: a prospective study." Nephron 51(4): 462-465.

Thaunat, O., C. Beaumont, et al. (2007). "Late introduction of sirolimus induces anemia in renal transplant recipients." Transplantation 83(9): 1283; author reply 1284.

Traindl, O., U. Barnas, et al. (1994). "Recombinant human erythropoietin in renal transplant recipients with renal anemia." Clinical transplantation 8(1): 45-48.

Turkowski-Duhem, A., N. Kamar, et al. (2005). "Predictive factors of anemia within the first year post renal transplant." Transplantation 80(7): 903-909.

van Dullemen, H. M., S. Luykx-de Bakker, et al. (1992). "Inhibitors of recombinant human erythropoietin in chronic renal failure." The Netherlands journal of medicine 41(12): 56-63.

Vanrenterghem, Y., C. Ponticelli, et al. (2003). "Prevalence and management of anemia in renal transplant recipients: a European survey." American journal of transplantation : official journal of the American Society of Transplantation and the American Society of Transplant Surgeons 3(7): 835-845.

Wizemann, V., R. Schafer, et al. (1993). "Follow-up of cardiac changes induced by anemia compensation in normotensive hemodialysis patients with left-ventricular hypertrophy." Nephron 64(2): 202-206.

Yorgin, P. D., J. D. Scandling, et al. (2002). "Late post-transplant anemia in adult renal transplant recipients. An under-recognized problem?" American journal of transplantation : official journal of the American Society of Transplantation and the American Society of Transplant Surgeons 2(5): 429-435.

Yoshimura, N., T. Oka, et al. (1989). "Cyclosporine-associated microangiopathic hemolytic anemia in a renal transplant recipient." The Japanese journal of surgery 19(2): 223228.

Zazgornik, J. (1997). "Azathioprine induced macrocytosis and red cell aplasia in renal transplant patients." Nephrology, dialysis, transplantation : official publication of the European Dialysis and Transplant Association - European Renal Association 12(3): 628 . 


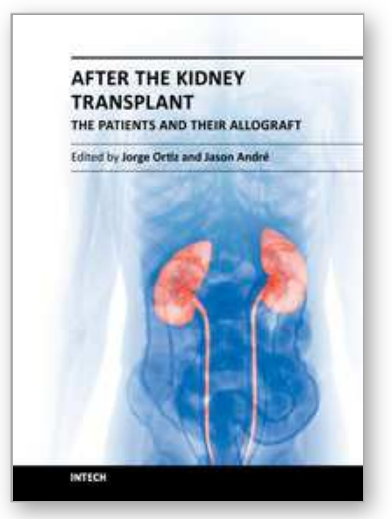

\author{
After the Kidney Transplant - The Patients and Their Allograft \\ Edited by Prof. Jorge Ortiz
}

ISBN 978-953-307-807-6

Hard cover, 386 pages

Publisher InTech

Published online 17, August, 2011

Published in print edition August, 2011

There are many obstacles in kidney transplantation. For the transplant team, there is the balance between immunosuppression to aid in the recipientâ $€^{\mathrm{TM}} \mathrm{S}$ tolerance of the allograft and the infection risk of a suppressed immune system. These potential long term complications of kidney transplantation are relatively well known, but there are many other complications that patients and families do not consider when preparing themselves for a kidney transplant. Although the benefits of attempting a kidney transplant far outweigh downfalls of the long term sequelae, kidney transplantation is by no means a benign procedure. It is the hope of these authors that the reader will leave with a sense of understanding towards the kidney recipients.

\title{
How to reference
}

In order to correctly reference this scholarly work, feel free to copy and paste the following:

Bhadran Bose and David Mudge (2011). Post Transplant Anaemia, After the Kidney Transplant - The Patients and Their Allograft, Prof. Jorge Ortiz (Ed.), ISBN: 978-953-307-807-6, InTech, Available from:

http://www.intechopen.com/books/after-the-kidney-transplant-the-patients-and-their-allograft/post-transplantanaemia

\section{INTECH}

open science | open minds

\section{InTech Europe}

University Campus STeP Ri Slavka Krautzeka 83/A 51000 Rijeka, Croatia Phone: +385 (51) 770447

Fax: +385 (51) 686166 www.intechopen.com

\section{InTech China}

Unit 405, Office Block, Hotel Equatorial Shanghai No.65, Yan An Road (West), Shanghai, 200040, China 中国上海市延安西路65号上海国际贵都大饭店办公楼405单元 Phone: +86-21-62489820

Fax: +86-21-62489821 
(C) 2011 The Author(s). Licensee IntechOpen. This chapter is distributed under the terms of the Creative Commons Attribution-NonCommercialShareAlike-3.0 License, which permits use, distribution and reproduction for non-commercial purposes, provided the original is properly cited and derivative works building on this content are distributed under the same license. 